\title{
Synthesis, Thermal Stability and the Effects of Ion Irradiation in Amorphous Si-O-C Alloys
}

Juan A. Colón Santana ${ }^{1}$, Elena Echeverría Mora ${ }^{2}$, Lloyd Price ${ }^{3}$, Robert Balerio ${ }^{3}$, Lin Shao $^{3}$ and Michael Nastasi ${ }^{4,5 *}$

${ }^{1}$ Department of Physics, Northern Illinois University, DeKalb, IL 60115

2 Department of Physics and Astronomy, University of Nebraska-Lincoln, Theodore Jorgensen Hall, 855 North 16th Street, Lincoln, NE 68588-0299, USA

3 Department of Nuclear Engineering, Texas A\&M University, 3133 TAMU, College Station, TX 77843-3133

${ }^{4}$ Nebraska Center for Energy Sciences Research, University of Nebraska-Lincoln, 230 Whittier, 2200 Vine Street, Lincoln, NE 68583-0857, USA

${ }^{5}$ Department of Mechanical and Materials Engineering, University of Nebraska-Lincoln, Lincoln, NE 68583-0857, USA

* Corresponding Author: mnastasi2@unl.edu

\begin{abstract}
Amorphous films of $\mathrm{Si}-\mathrm{O}-\mathrm{C}$ alloys were synthesized via sputtering deposition at room temperature. These alloys were characterized using grazing incidence diffraction, both as a function of temperature and irradiation dose. It was found that the material retained its amorphous structure, both at high temperatures (up to $1200^{\circ} \mathrm{C}$ ) and ion irradiation doses up to $1.0 \mathrm{dpa}$. The depth profile from photoemission spectroscopy provided evidence of the oxidation state of these alloys and their atomic composition. The studies suggest that Si-O-C alloys might belong to a group of radiation tolerant materials suitable for applications in reactor-like harsh environments.
\end{abstract}


Keywords: Sputtering Deposition, Amorphous Materials, X-ray Diffraction, X-ray Photoemission, Ion Irradiation

\section{Introduction}

The effects of radiation damages in crystalline materials have been studied for decades. It has been established that the primary form of radiation damage in this type of materials are point defects formed during the collision cascade events, when an energetic particle interact with the atoms in the target material [1]. Under ion or neutron irradiation, supersaturated point defects resulting from damage cascade creation cannot be completely removed through defect recombination. A small fraction of these defect develop into extended defect clusters such as voids, dislocations and dislocation networks, leading to changes in the dimension of the material, causing property degradation. The development of radiation tolerant materials for high performance nuclear reactors calls for innovative approaches to increase self-defect-repairing capabilities. Grain and interphase boundaries in metals have been shown to act as defect sinks (i.e. defects migrate and are trapped at the boundaries) [2,3], thus, radiation damages can be reduced, prolonging the structural stability (and property sustainability) of the material [4,5].

Unlike crystalline solids, the fundamental processes by which amorphous alloy/crystalline composites respond to steady-state irradiation have received relatively less attention [6] and are still largely unknown. One key issue regarding a description of radiation damages in amorphous materials is whether point defects are formed after the radiation event take place $[7,8]$. Moreover, competing atomic mechanisms have been proposed to account for the macroscopic effects induced by radiation, in which the role of a point defect is either acknowledge or eluded $[9,10]$. Nonetheless, it is assumed that amorphous materials possess no translational symmetry, therefore, do not contain conventional crystal defects such as vacancies, 
interstitials, or dislocations [11]. If such is the case, what are the effects of radiation in amorphous materials? Some amorphous alloys could be radiation indifferent within an envelope of irradiation conditions. In certain amorphous alloys the radiation-induced damage may anneal out faster than it is created, allowing these alloys to persist indefinitely in an externally driven steady-state with time-invariant structure and properties. For such alloys, no point defects or defect clusters are formed, as oppose to its crystalline counterpart, instead free volume is generated as a local disturbance. Thus, the radiation-induced damage may anneal out without the need for trapping or recombination of defects (such as vacancies and interstitials). These materials may offer the possibility of eliminating the root cause responsible for radiation damage in polycrystalline solids (namely the production of point defects and clusters thereof in collision cascades) and may serve as the basis for developing a new class of structural materials with unprecedented resistance to radiation.

We speculate that the irradiation resistance of an amorphous alloy may be closely coupled to its crystallization temperature. For this reason, a $\mathrm{Si}-\mathrm{O}-\mathrm{C}$ alloy was selected. Previous work on silicon oxycarbide glasses provide evidence of structural stability to $1000-1200^{\circ} \mathrm{C}$ in argon and in air environment $[12,13,14]$. The very high crystallization temperature of these amorphous-ceramics suggests these will be resistant to crystallization under irradiation to high temperatures. The aim of this work is to study the thermal stability and irradiation response at room temperature of an amorphous Si-O-C alloy layer of different alloy compositions.

\section{Experimental}

The amorphous Si-O-C alloys were grown using the ATC-2000F sputtering system (AJA International). The alloys were deposited on a silicon substrate oriented along the (100) crystallographic direction and with a thermalized $\mathrm{SiO}_{2}$ layer of around $3000 \AA$, employed to 
minimize the diffusion of the alloy content onto the substrate side at high temperatures. Prior to deposition, the substrates were cleaned via sonication, first in an acetone bath, followed by submersion in methanol, for a total of $10 \mathrm{~min}$ in each solution. The alloy depositions were carried via co-sputtering of an amorphous silicon carbide ( $\mathrm{SiC}$ ) and an amorphous silicon dioxide $\left(\mathrm{SiO}_{2}\right)$ target. Due to the strong insulating nature of these target materials (particularly $\mathrm{SiO}_{2}$ ), two radio frequency (RF) magnetron sputtering guns were employed for each target material to minimize the charging effects present in both targets. The base pressure of the ultrahigh vacuum chamber was in the upper $10^{-8}$ Torr prior deposition. The Si-O-C alloys were synthesized at an ambient temperature with an argon pressure of 5.0 mTorr and a sample rotation of $70 \mathrm{rpm}$. The alloy composition was controlled by varying the power supplied to the sputtering guns. The power values were dictated by a preliminary calibration plot (Sputtering rate vs Power). Since the targets employed were poor thermal conductors, the sputtering rate values for both targets were maintain at a power range ( $25 \mathrm{~W}-55 \mathrm{~W}$ ) considered safe to avoid (or minimize) damages to the target in case of an abrupt shutdown of the system (such as plasma failure). The thickness were calculated to satisfy the sputtering ratio of 1:1, 1:2 and 2:1 alloys, where $\mathrm{x}: \mathrm{y}$ refers to the $\mathrm{SiC}: \mathrm{SiO}_{2}$ sputtering ratio. The sputtered ratios were smaller than those observed by Du Ping (et. al.)[15] in which the same deposition method was used.

The thicknesses of the alloys were measured using a Dektak-xp Stylus Profilometer and confirmed within $5.0 \%$ error with a thin film thickness monitor. Each alloy thickness value was based on the average of five consecutive measurements spanning different spatial locations along the film step. The values are recorded in Table 1. 


\begin{tabular}{|c|c|}
\hline $\begin{array}{c}\text { Sputtering Ratio } \\
\left(\mathbf{S i C} \text { to } \mathbf{S i O}_{2}\right)\end{array}$ & Thickness $(\mathbf{\AA})$ \\
\hline $1: 1$ & 3380 \\
\hline $1: 2$ & 3384 \\
\hline $2: 1$ & 3157 \\
\hline
\end{tabular}

Table 1 - The alloy thickness as obtained by profilometry measurements based on the SiC to $\mathrm{SiO}_{2}$ targets sputtering ratio.

The annealing of the Si-O-C alloys was performed using a Lindberg 55322 oven with a quartz tube under vacuum conditions. The base pressure prior the annealing cycle was on the low $10^{-6}$ Torr. The ramping time for the onset temperature was 30 minutes, with an overnight cooling cycle down to room temperature.

Samples were irradiated by using $100 \mathrm{keV}$ He ions to a maximum displacement per atom (dpa) value of $1.0 \mathrm{dpa}$ (corresponding to $1.1 \times 10^{17} \mathrm{ions} / \mathrm{cm}^{2}$ ) at an ion flux of $\sim 2 \times 10^{13}$ ions $/ \mathrm{cm}^{2} / \mathrm{sec}$ at temperatures up to $600{ }^{\circ} \mathrm{C}$. The use of $100 \mathrm{keV} \mathrm{He}$ was chosen to ensure that that no He ion implantation occurred in the Si-O-C film and that the film only received displacement damage. SRIM simulations [16] showed that the range of the $100 \mathrm{keV} \mathrm{He}$ was $700 \mathrm{~nm}$, far from the Si-O-C layer. In addition, experiments have shown that light ion irradiation provides a good emulation of neutron damage in materials [17]. During the ion irradiation, the temperature was monitored by thermal couples attached to the sample holder surface, and used as feedback for automatic temperature control system. The temperature variation during the irradiation was less than $5^{\circ} \mathrm{C}$. The base pressure during irradiation was better than $5 \times 10^{-5}$ torr. A static defocused beam of $1.0 \mathrm{~cm}$ in diameter was used, to avoid any void suppression from a pulsed beam when beam rastering was applied.

For the structural studies, grazing incidence x-ray diffraction (GIXRD) with parallel beam geometry was employed using a Bruker D8 Diffractometer. This configuration minimized 
the substrate contribution to the spectra, while enhancing the features at the outermost layers (if not the entire layer) of the Si-O-C alloy films. Prior to each particular measurement, the specimen was aligned accordingly and data was collected in the detector scan mode.

The Si-O-C alloys chemical state and composition was determined using X-ray photoemission spectroscopy. The $\mathrm{Mg}-\mathrm{K} \alpha$ line $(1253.6 \mathrm{eV})$ was used and photoelectrons with a passing energy of $50 \mathrm{eV}$ were detected. The photoelectrons were collected normal to the surface of the film, unless otherwise stated. Since photoemission is a surface sensitive technique (probing depth 15-20 ), the composition is strictly restricted to the outermost layers of the material. However, a bulk composition study was performed by exposing the material to an ionize argon beam $\left(\mathrm{Ar}^{+}\right.$pressure $\sim 10^{-5}$ Torr) at intervals of 10 minutes for a total of an hour. To maximize the uniformity of the reduced surface layer, the samples were rotated $\pm 40^{\circ}$ off with respect to the incident ion beam during each sputtering cycle. Data was collected immediately after each sputtering cycle. The composition values are based on the average of all the collected data sets. Charging effects were observed and corrected by a shift in binding energy based on the expected $\mathrm{O} 1 \mathrm{~s}$ core level binding energy and confirmed with the use of an electron flood gun. For the atomic composition calculation, the proportionality constant between the corresponding ratios was calculated using a $\mathrm{SiC}$ and $\mathrm{SiO}_{2}$ standard crystal. For each core level feature, the background was modeled and subtracted using a Shirley type function and the area under the peak was obtained based on the fit of a Gaussian distribution function.

\section{Thermal Stability of the Si-O-C Amorphous Alloys}

The structural characterization of the alloys was performed using grazing incidence X-ray diffraction (GIXRD). To study the thermal stability of the alloys, the samples were annealed under vacuum condition at different temperatures as shown in Figure 1. 


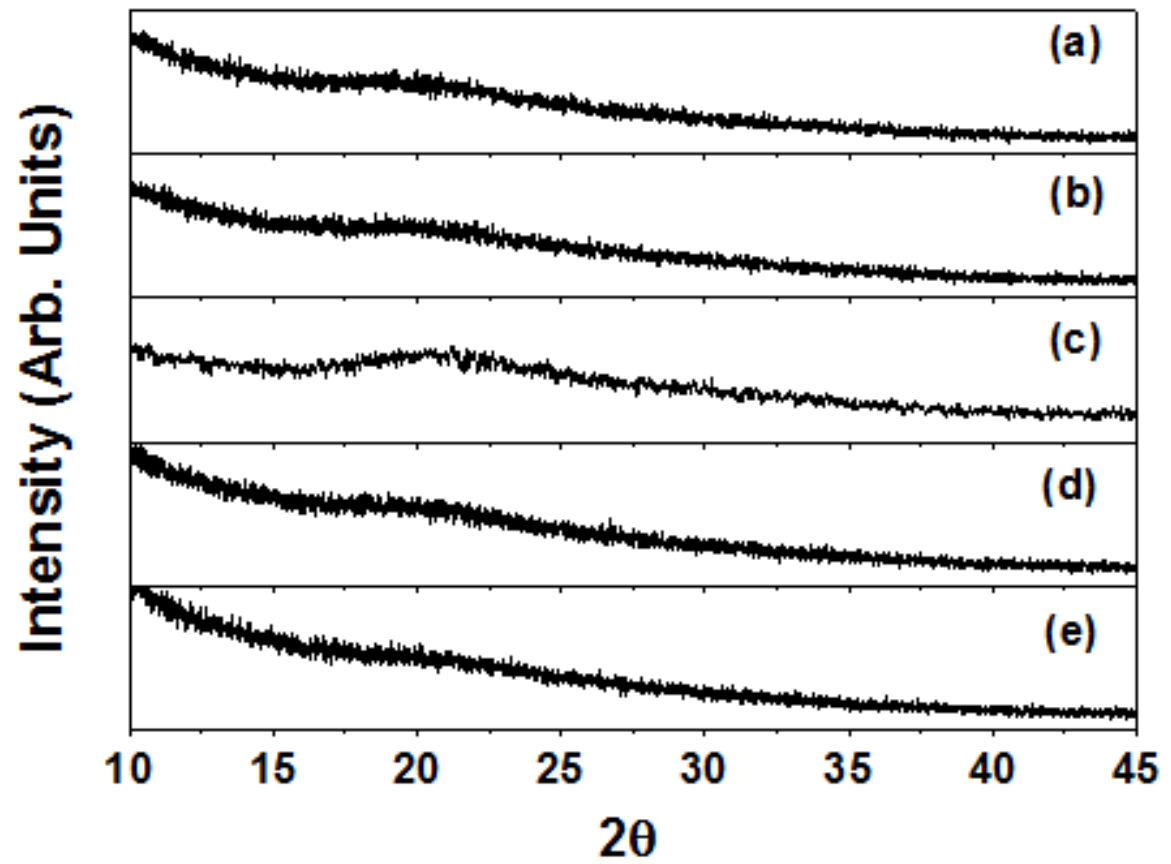

Figure 1 - Shows the grazing incidence $\mathrm{x}$-ray diffraction spectra obtained at temperatures of (a) $23{ }^{\circ} \mathrm{C}$, (b) $250{ }^{\circ} \mathrm{C}$, (c) $500{ }^{\circ} \mathrm{C}$, (d) $750{ }^{\circ} \mathrm{C}$ and (e) $1000^{\circ} \mathrm{C}$ for the $1: 1 \mathrm{Si}-\mathrm{O}-\mathrm{C}$ alloy. The spectra was taken at incidence angle and detector scan mode.

The broad feature at around $21.0^{\circ}$ provides evidence of the amorphous character of the alloy and is strongly weighted to the $\mathrm{Si}-\mathrm{O}$ bonding in the amorphous phase. These results are consistent with those seen [14] when the oxygen content in the alloy is greater than that of Carbon. Similar results were also observed by [18], when the material was grown via a polymer precursor method. No crystallographic peak or any other feature were observed in our films along the $2 \theta$ range of $90^{\circ}$ for temperatures up to $1200^{\circ} \mathrm{C}$ (not shown) and annealing times of 2.0 hours. This is consistent with the expected crystallization temperatures found by Jeone (et.al.)[16] and Domenico (et. al.) [19]. A small shift of the broad peak towards a higher $2 \theta$ value was observed and is shown in Figure 2. This suggests that the atomic structure undergoes an atomic relaxation as the temperature increases. Nonetheless the onset of crystallization is not observed. Similar 
trends (no crystalline phases) were observed in the other $\mathrm{Si}-\mathrm{O}-\mathrm{C}$ alloys (i.e. 1:2 and 2:1 $\mathrm{SiC}_{-} \mathrm{SiO}_{2}$ sputtering rates) for temperatures up to $1200^{\circ} \mathrm{C}$.

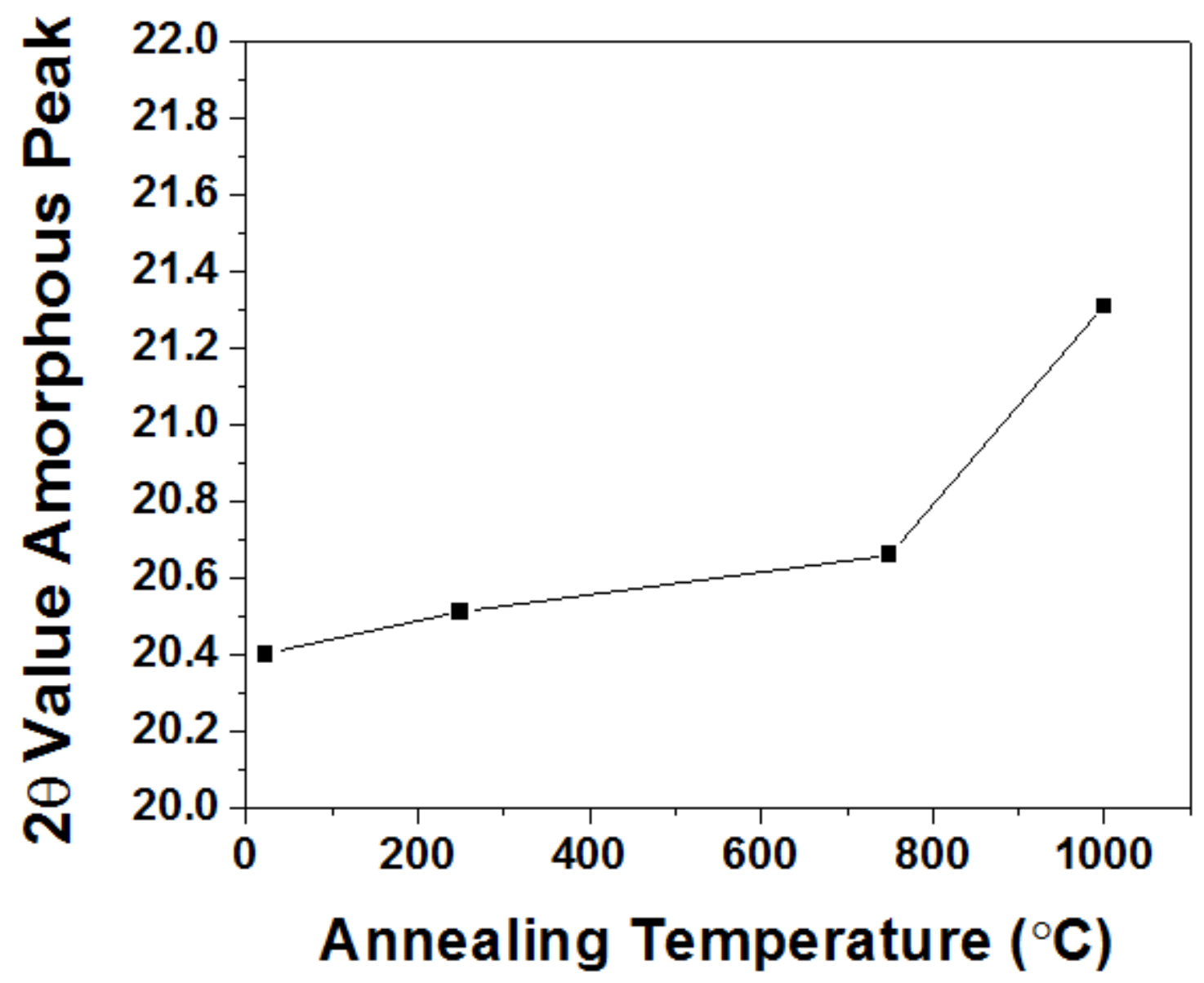

Figure 2 - Shows the amorphous peak shift extracted from the GIXRD data as a function of temperature as obtained for the Si-O-C alloy synthesized with a sputtering ratio 1:1.

$\mathrm{X}$-ray photoemission measurements were done on the Si-O-C alloys at room temperature and the results are shown in Figure 3. Both, the $\mathrm{C} 1 \mathrm{~s}$ and $\mathrm{O} 1 \mathrm{~s}$ core level features are found at a fixed binding energy of $284.4 \mathrm{eV}$ and $532.2 \mathrm{eV}$ respectively for all alloys compositions. The $\mathrm{Si}$ $2 \mathrm{p}$ core level spectra show a shift in binding energy that can be correlated to the amount of oxygen content in the alloy. For the alloy with a sputtering rate of $2: 1$, the binding energy was found at $102.7 \mathrm{eV}$, followed by the $1: 1$ alloy at $103.1 \mathrm{eV}$ and $103.5 \mathrm{eV}$ for $1: 2$. While the core 
level binding energy assignment of $\mathrm{SiC}$ and $\mathrm{SiO}_{2}$ phase is somewhat straightforward [20], the assignment of a chemical state in these type of alloys is very complex. In fact, there are five known different species that can be found in a Si-O-C compound as shown in Table 2.

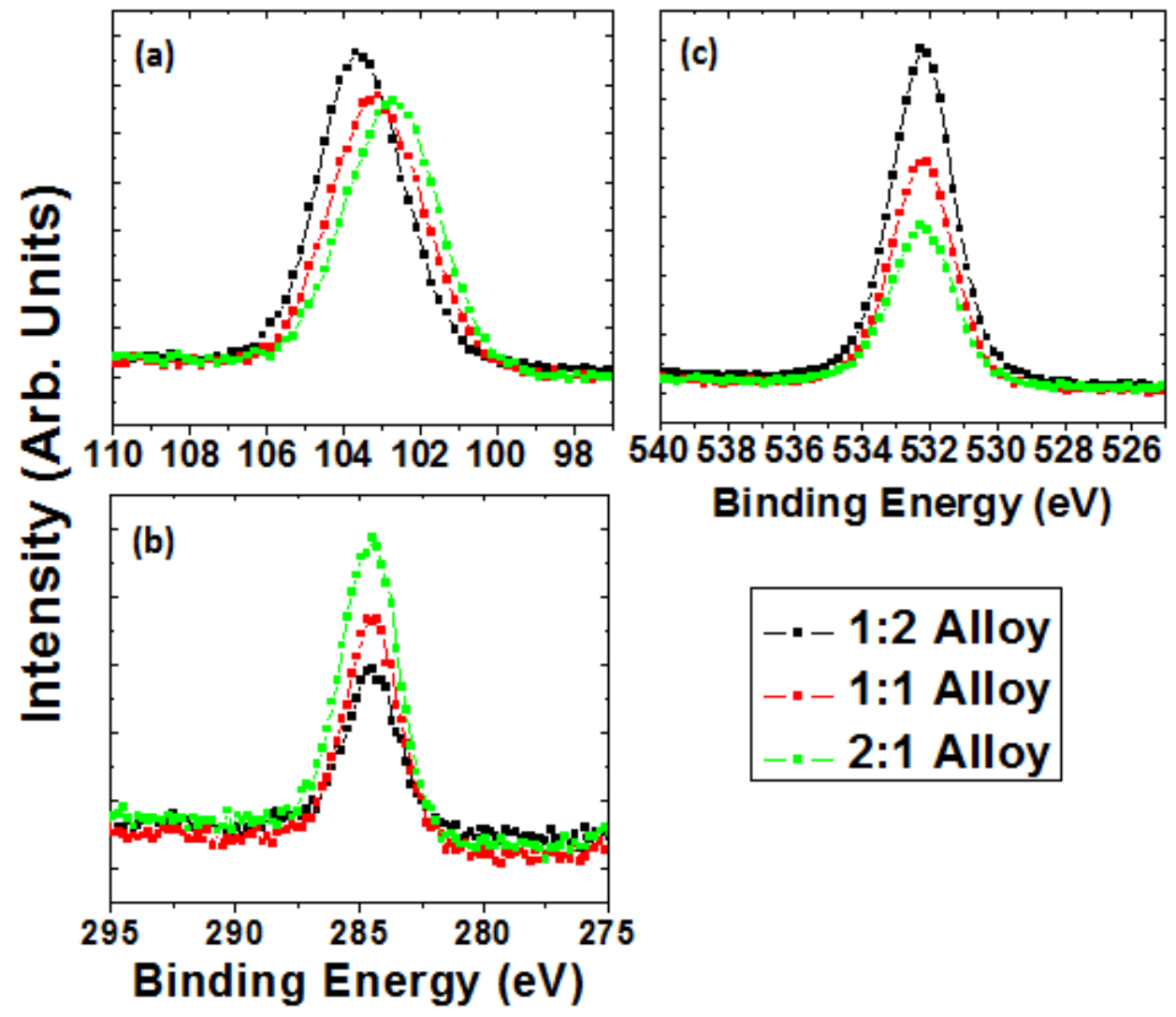

Figure 3 - The core level spectra after the $5^{\text {th }}$ sputtered clean cycle for (a) Si 2p, (b) C 1s and (c) $\mathrm{O} 1 \mathrm{~s}$ features at room temperature. The alloys films were annealed under vacuum conditions for $60 \mathrm{~min}$ for each temperature and charging effects were referenced to the expected $\mathrm{O} 1 \mathrm{~s}$ signal.

\begin{tabular}{|c|c|c|c|c|}
\hline \multirow{2}{*}{$\begin{array}{l}\text { Si-O-C } \\
\text { Species }\end{array}$} & \multicolumn{4}{|c|}{ Binding Energies (eV) } \\
\hline & \multicolumn{2}{|c|}{ Si 2p } & $\mathrm{C} \mathrm{1s}\left[{ }^{21}\right]$ & O 1s[20] \\
\hline $\mathrm{SiC}_{4}$ & $100.2-100.4$ & & & \\
\hline $\mathrm{SiOC}_{3}$ & $\begin{array}{c}101.0-101.2 \\
{[21]}\end{array}$ & $\begin{array}{c}100.2-100.3 \\
{[20]}\end{array}$ & 283.7 [20] & $532.2[\mathbf{2 0}]$ \\
\hline $\mathrm{SiO}_{2} \mathrm{C}_{2}$ & $\begin{array}{c}101.8-102.0 \\
{[21]}\end{array}$ & $\begin{array}{c}101.2-101.6 \\
{[20]}\end{array}$ & $283.7[20]$ & $532.2[\mathbf{2 0}]$ \\
\hline $\mathrm{SiO}_{3} \mathrm{C}$ & $\begin{array}{c}103.4-103.6 \\
{[21]}\end{array}$ & $\begin{array}{c}102.1-102.2 \\
{[20]}\end{array}$ & $283.7[\mathbf{2 0}]$ & $532.2[\mathbf{2 0}]$ \\
\hline$\overline{\mathrm{SiO}_{4}}$ & $\begin{array}{c}102.6-102.8 \\
{[21]}\end{array}$ & & & \\
\hline
\end{tabular}


Table 2 - The species and core level binding energies expected in a Si-O-C alloy film.

In some instances (provided enough resolution), these phases can be distinguished as separate peaks [28], in other cases a deconvolution of a broad peak (a few eV's wide) has been attempted $[23,29]$. None of these two results were observed here. The width of the Si $2 p$ core level peak is $2.1 \mathrm{eV}$ (only $0.3 \mathrm{eV}$ wider than that corresponding to the $\mathrm{Si}-\mathrm{Si}$ bond in a Si crystal) with no shoulder or other visible contribution to the spectra. This made the assignment of the chemical state complex and an attempt to deconvolute the spectra will likely provide results with very large uncertainties on the attributed species. In this study, the Si-O-C alloys were treated as a single phase alloy with a chemical shift proportional to the oxygen content in the material. The changes in intensity of the core level (or peak area) are proportional to the atomic content of the specific element in the alloy. This indeed is consistent with the concentration expected from the stoichiometry of the target material under ideal deposition conditions (i.e. the 2:1 alloy should have a higher $\mathrm{C}$ content and the $1: 2$ should have a higher oxygen content in reference to the $1: 1$ alloy composition).

The Si-O-C alloys were annealed under a vacuum in the upper $10^{-6}$ Torr for 60 minutes at different temperatures. The core level spectra after vacuum annealing is shown in Figure 4 for the Si-O-C alloy synthesized via co-sputtering with a $1: 1 \quad \mathrm{SiC}_{\mathrm{SiO}}$ target ratio. There are no major changes in the chemistry at the alloy surface as suggested by the invariant location of the core level peaks for each atomic orbital. No additional phases are observed after the annealing cycles. The relative intensities are not altered after the annealing suggesting that little changes have occur in the Si-O-C alloy composition has occurred in the alloy. The compositions calculated for the 1-1 Alloy as a function of temperature is shown in Figure 5. 


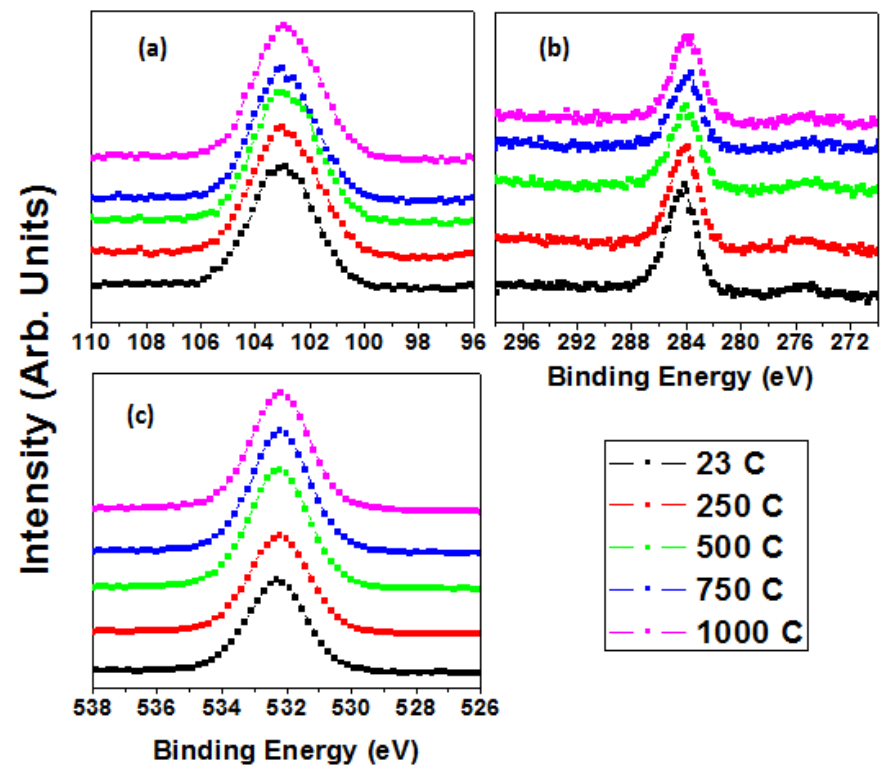

Figure 4 - The core level photoemission spectra for (a) Si 2p, (b) C 1s and (c) O 1s electronic orbital as a function of temperature obtained for the Si-O-C alloy synthesized with a cosputtering rate of 1:1. The alloys films were annealed under vacuum conditions for $60 \mathrm{~min}$ for each temperature and charging effects were referenced to the expected $\mathrm{O}$ 1s signal. Photoelectrons were collected normal to the surface. The spectra correspond to that after the $5^{\text {th }}$ $\mathrm{Ar}^{+}$sputtered cycle.

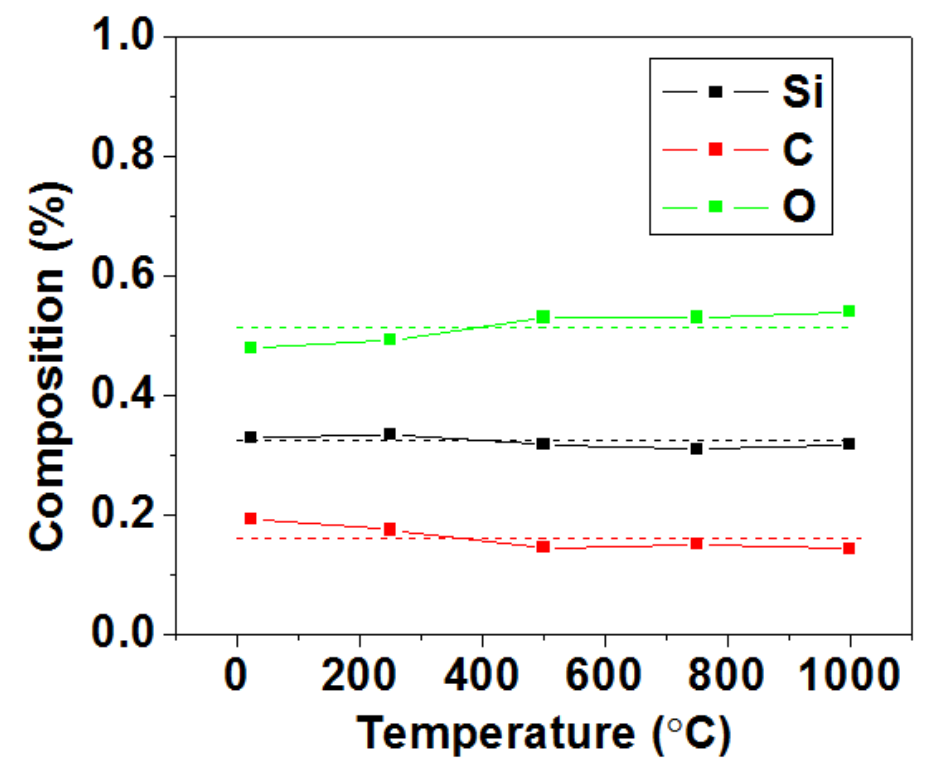

Figure 5 - The atomic composition obtained from the core level photoemission spectra for the Si-O-C alloy synthesized with a co-sputtering rate of $1: 1$ as a function of temperature. The dashed lines represent the average concentration as a function of sputtering depth. 
As stated earlier, each core level peak data set was properly reduced and fitted with the corresponding profile. The dashed line represents the average composition of the alloy as a function of temperature. Table 3 summarized the compositions obtain for the alloys as a function of sputtering depth as grown (no annealing). Note an oxygen rich alloy seems to dominate, even when the sputtering rate of $\mathrm{SiC}$ was twice that of $\mathrm{SiO}_{2}$.

\begin{tabular}{|c|c|c|c|}
\hline $\begin{array}{c}\text { Sputtering Ratio } \\
\left(\mathbf{S i C} \text { to } \mathrm{SiO}_{2}\right)\end{array}$ & \multicolumn{3}{|c|}{ Composition (\%) } \\
\cline { 2 - 4 } & $\mathbf{S i}$ & $\mathbf{C}$ & $\mathbf{O}$ \\
\hline $1: 1$ & 31.0 & 14.0 & 55.0 \\
\hline $1: 2$ & 31.0 & 10.0 & 59.0 \\
\hline $2: 1$ & 33.0 & 27.0 & 40.0 \\
\hline
\end{tabular}

Table 3 - The average atomic composition of the amorphous Si-O-C alloy as a function of sputtering depth.

\section{Correlation between the Free Volume and Charging Effects in XPS}

During the photoemission process, the specimen is maintained in electrical contact to a grounded electrode. This ensures that each photoelectron (electron emitted from the sample surface) is promptly compensated and no net charge is built within the probed volume of the material. In insulating materials, like $\mathrm{Si}-\mathrm{O}-\mathrm{C}$ alloy, there are not many free electrons to recombine with the holes generated by the photoemission process and those available do not travel fast enough for recombination to occur at a faster rate. This will result in a region of positive charge within the probe volume that can interact electrically with the photoelectron prior its release from the specimen. This may lead to undesirable effects in the core level photoemission spectra (peak distortions, shifts, or both). Charging can be divided in several categories depending on the effect seen in the core level feature. For the Si-O-C alloys a distortion of the core level spectra was absent (i.e. no change in width, intensity or shape was observed), only a shift was present. Such behavior suggests the development of a homogeneous 
potential at the probed volume of the films. For an electric potential with such properties, the photoemission spectra can be corrected by a shift in the core level spectra by a constant value (as determine by a reference peak, adventitious carbon being typical). For this reason, the spectra's were corrected by shifting the $\mathrm{O} 1 \mathrm{~s}$ core level signal to the expected value of $532.2 \mathrm{eV}$ without any loss of information. The absence of an electron tail into the lower binding energy regime and the similarity in intensity [24] provide evidence of the reliability of this data. While charging effects are usually undesirable, certain scenarios allows for their exploitation in benefit of the study being done [25].

In crystals, vacancies are known to act as donors at room temperature [26,27]. In analogy, the changes in binding energy of the core level feature due to charging effects may be due to a decrease in free volume of the amorphous alloys. In fact several studies have been done that relate the conductivity of a glass to the free volume [28]. Since the deposition of the Si-O-C alloy was carried out at room temperature, uncompensated bonding (or weak bonding) are likely to form, as the amplitudes of the atomic vibrations are somehow limited at such temperature. At high temperatures, the atomic vibrations are large enough that the atoms can rearrange themselves into a more stable configuration (although still non-crystalline). If such is the case, the atomic bonding between the atoms is expected to strengthen, affecting the already low conductivity inherent in the alloy. Figure 6 shows the binding energy of the Si $2 p$ peak as a function of temperature with no charge correction. 

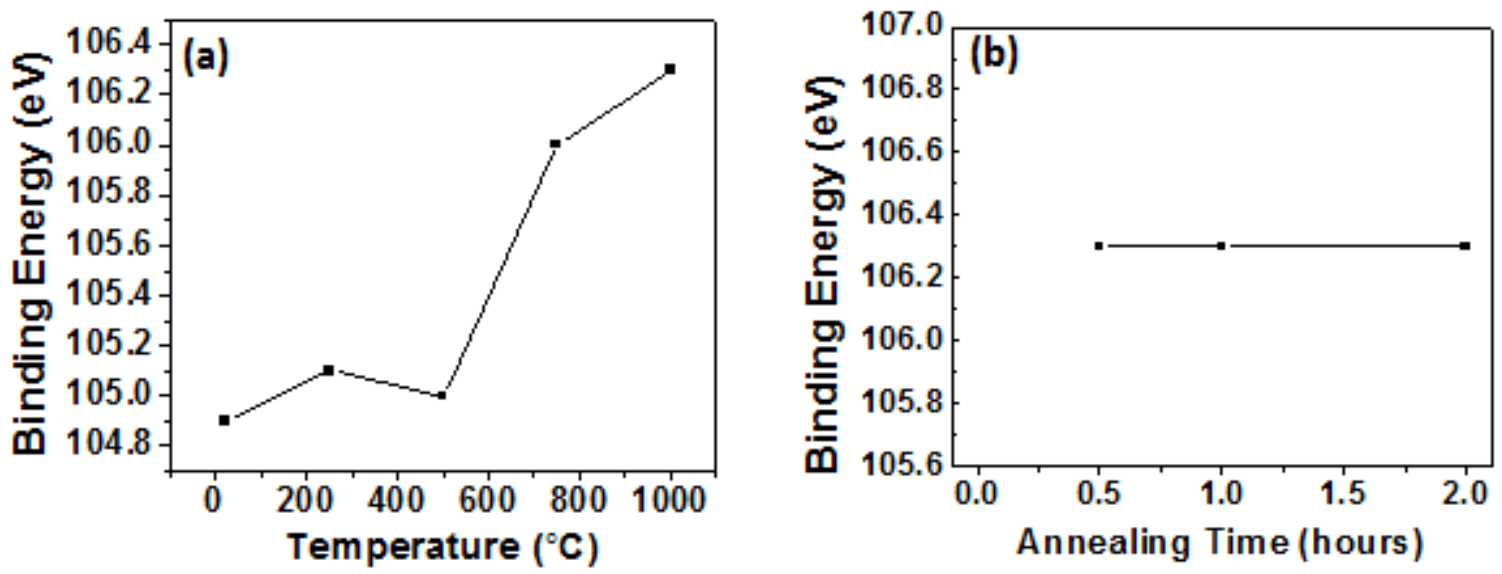

Figure 6 - The Si $2 p$ core level peak as a function of (a) temperature and (b) annealing time at $1000^{\circ} \mathrm{C}$ for the $\mathrm{Si}-\mathrm{O}-\mathrm{C}$ alloy synthesized with a co-sputtered ratio of $1: 1$ without charging correction to the XPS spectra.

As the temperature increases, the Si $2 p$ core level peak shifts into a higher binding energy. The observed core level shift is not related to a change in chemical composition in the alloy, but may be due to a decrease in conductivity (or free volume) in the alloy itself, consistent with the structural data in Figure 2. Several annealing cycles were done at $1000{ }^{\circ} \mathrm{C}$, and no further shifts in the core level peak were found. To further validate our claim that the behavior observed may be due a decrease in free volume as a function of temperature, and the effect shown in Figure 6 (a) is not a product of oxidation in the Si-O-C alloy, let us refer to Figure 7. If charging effects are responsible for the shift observed in the $\mathrm{Si} 2 \mathrm{p}$ core level peak, the differences in binding energies between the $\mathrm{Si} 2 \mathrm{p}$ core level and the $\mathrm{O} 1 \mathrm{~s}$ level must remain invariant as the temperature is increased. This is the case as shown in Figure 7. 


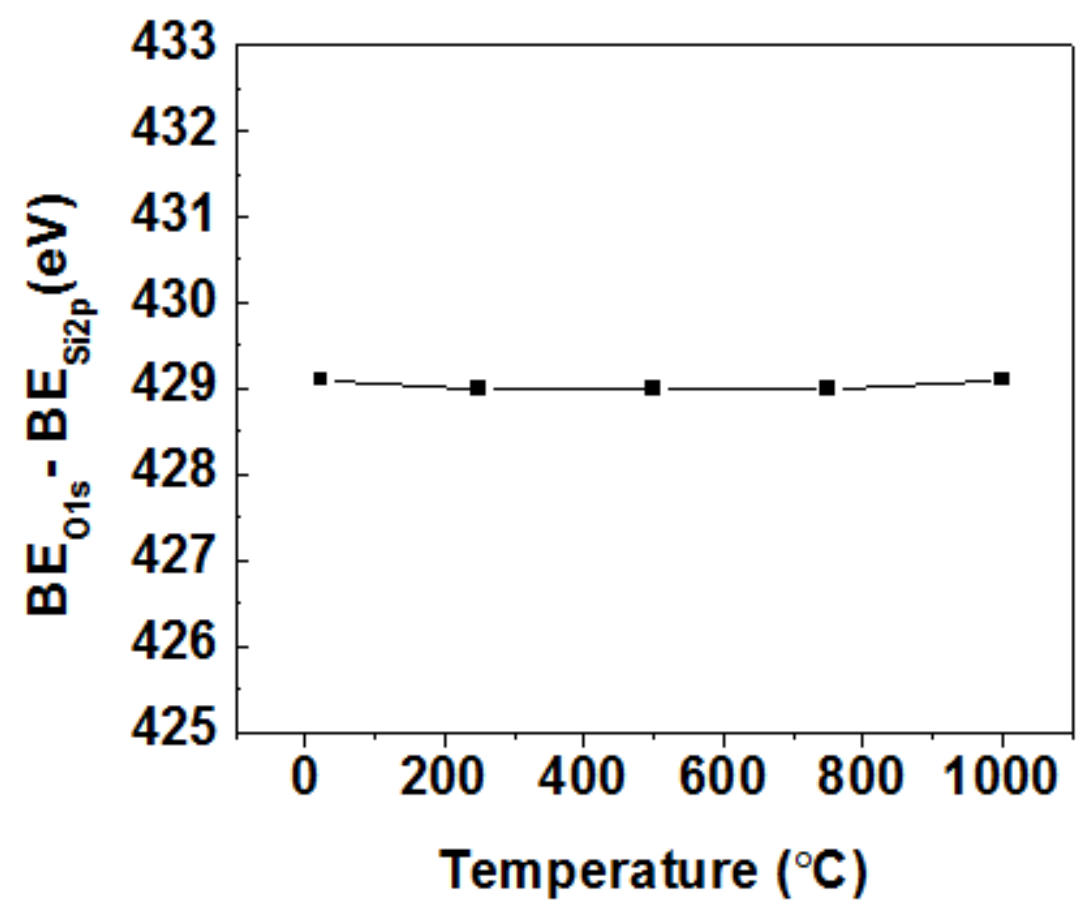

Figure 7 - The core level peak difference of the $\mathrm{O} 1 \mathrm{~s}$ and $\mathrm{Si} 2 \mathrm{p}$ orbitals as a function of temperature for the Si-O-C alloy synthesized with a co-sputtered ratio of 1:1. No corrections were applied to the observed charging effects.

\section{Structural Characterization and Core Level Spectroscopy after Ion Irradiation}

One common effect of radiation damages in crystals is the observation of an amorphous phase after irradiation $[29,30]$. In some amorphous materials, the reverse process of crystalline-toamorphous transitions might be challenging considering that crystallization of a disordered arrangement requires a high degree of correlated atomic movements due to its directional bonding nature. As previously described, such effects are likely due the inability of the material to restore the atomic rearrangement after the damage cascade and the clustering of point defects. In an amorphous material, crystallization may occur along the ion track. As the energetic ion begins forming the damage cascade, the surrounding atoms are perturbed creating disorder (in the already disordered material) along the ion tracks. This energy is subsequently dissipated in a thermal spike (a pocket of hot, liquid-like material several nanometers in diameter). Within 
picoseconds, the excess energy in this pocket is conducted away and the perturbed region returns to thermal equilibrium. Since no conventional point defects or defect clusters can be identified after a collision cascade in an amorphous alloy, atoms with increased excess free volume $(\Delta \mathrm{V}$ excess) are observed.

The Si-O-C alloys were exposed to ion irradiation and were studied using the GIXRD technique, as described and done previously, and no crystallographic phase was found.

In some cases, X-ray photoemission can detect radiation damages induced changes. If the incident ion is energetic enough as to displace the atoms vigorously, not only an increase in free volume is expected (in an amorphous film), but new bonding within surrounding atoms can form. Such interactions can result in either additional peaks at different binding energies or intensity changes in the pre-existing core level signal. In fact, photoemission signal changes from ion irradiated crystalline materials have been observed [31], and we expect some signal changes to be observed in ion irradiated amorphous materials if radiation damages occurred. X-ray photoemission data suggest that the material remain invariant under ion irradiation as shown in Figure 8 . The core level signal remains essentially the same during irradiation as compare to the signal of the virgin alloy. The composition shows little changes as shown in Figure 9. This seems reasonable (especially at room temperature) considering that crystallization of ceramics requires a high degree of correlated atomic movements due to its directional bonding nature. 


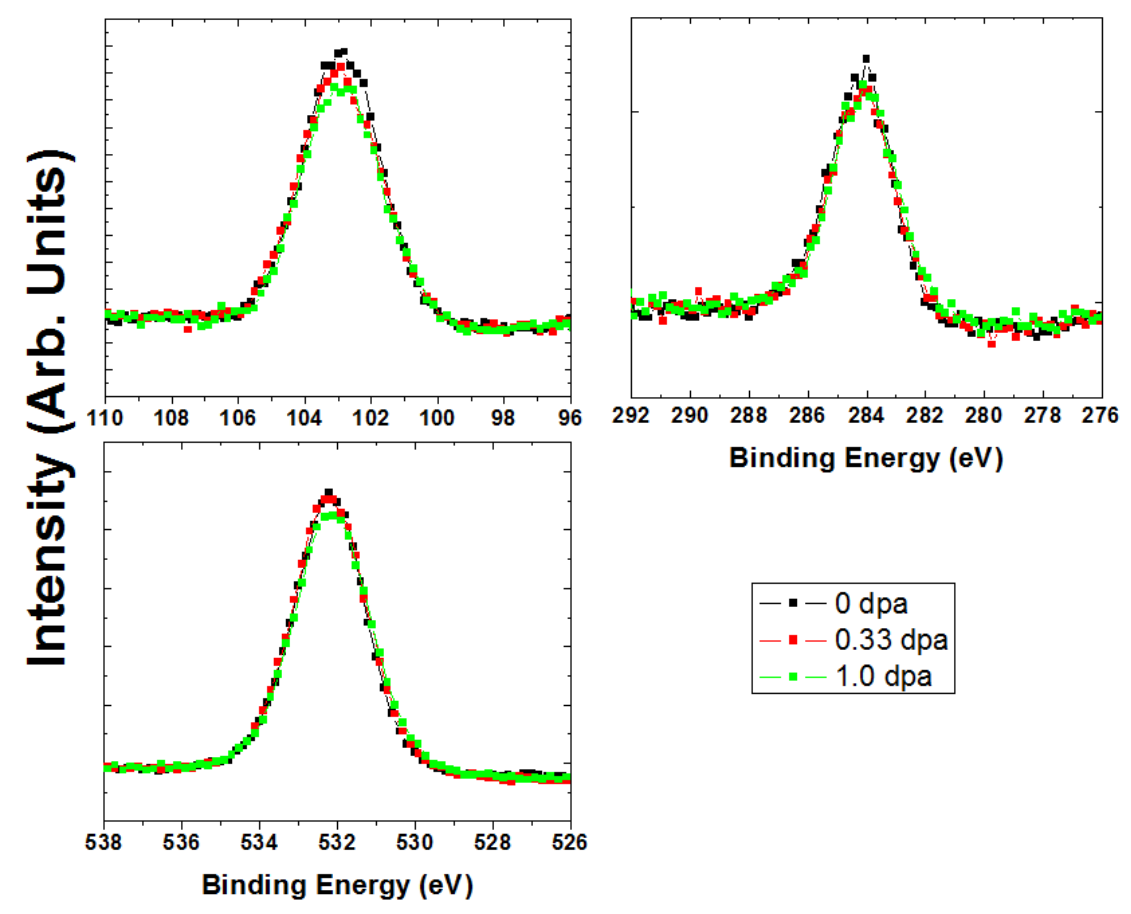

Figure 8 - The core level photoemission spectra for (a) Si 2p, (b) C 1s and (c) O 1s electronic orbital as a function of $\mathrm{He}^{+}$dose for the Si-O-C alloy synthesized with a co-sputtering rate of 1:1. Charging effects were referenced to the expected $\mathrm{O}$ 1s signal. Photoelectrons were collected normal to the surface. The spectra correspond to that after the $5^{\text {th }} \mathrm{Ar}^{+}$sputtered cycle.

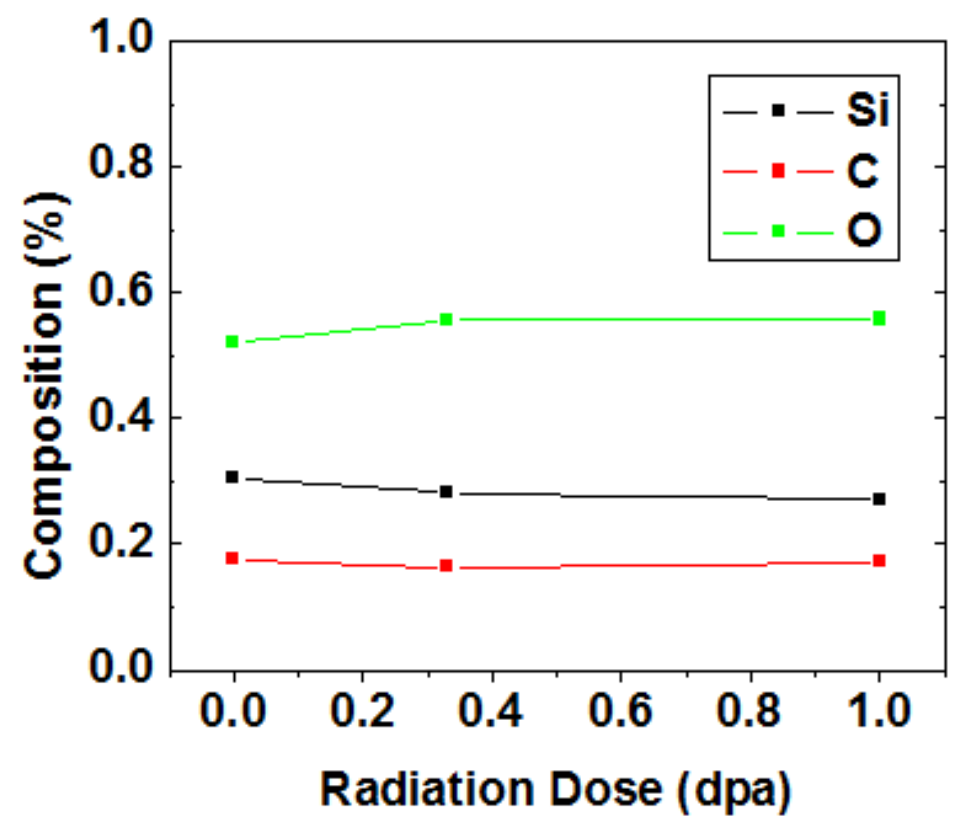

Figure 9 - The atomic composition obtained from the core level photoemission spectra for the Si-O-C alloy synthesized with a co-sputtering rate of 1:1 as a function of $\mathrm{He}^{+}$dose. 
The quenching rate can be significantly reduced to allow possible crystallization along ion tracks if the substrate is heated at elevated temperatures. The broad peak corresponding to the amorphous Si-O-C structure is barely evident, but the lack of sharp peaks suggests that the alloy did not undergo crystallization, even under hot irradiation. While the assignment of a peak is rather difficult (perhaps even meaningless), it seems that the ion irradiation does little in terms of a relaxation mechanism. Similar trends (no crystallization) were observed for the alloys cosputtered at a rate $1: 2$ and $2: 1$. It is suggested that the material is transparent to the ion irradiation.

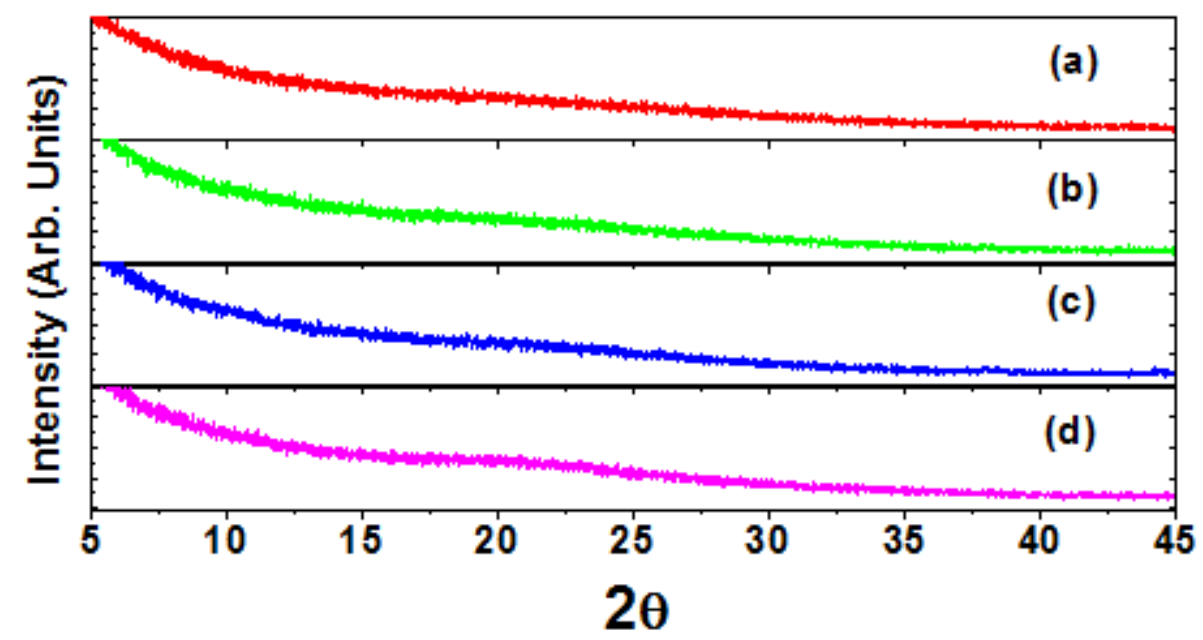

Figure 10 - The $\mathrm{x}$-ray diffraction spectra obtained for a $\mathrm{He}^{+}$irradiation of $1.0 \mathrm{dpa}$ at temperatures of (a) $23{ }^{\circ} \mathrm{C}$, (b) $200{ }^{\circ} \mathrm{C}$, (c) $400{ }^{\circ} \mathrm{C}$ and (d) $600{ }^{\circ} \mathrm{C}$ for the Si-O-C alloy synthesized with a co-sputtering ratio of 1:1. The spectra were taken at grazing incidence angle and in the detector scan mode. 
The Si-O-C X-ray photoemission data suggest that effects due to irradiation are negligible even at elevated temperatures. As shown in Figure 11, the chemical species of the alloy remain invariant after ion irradiation. No major changes in composition were observed as shown in Figure 12. This study suggest that the quenching rate of thermal spikes associated with damage cascade creation and diffusion of excessive free volume created along the ion track is faster than the relaxation time required for such crystallization, allowing the alloy to "self-heal" from the radiation event.

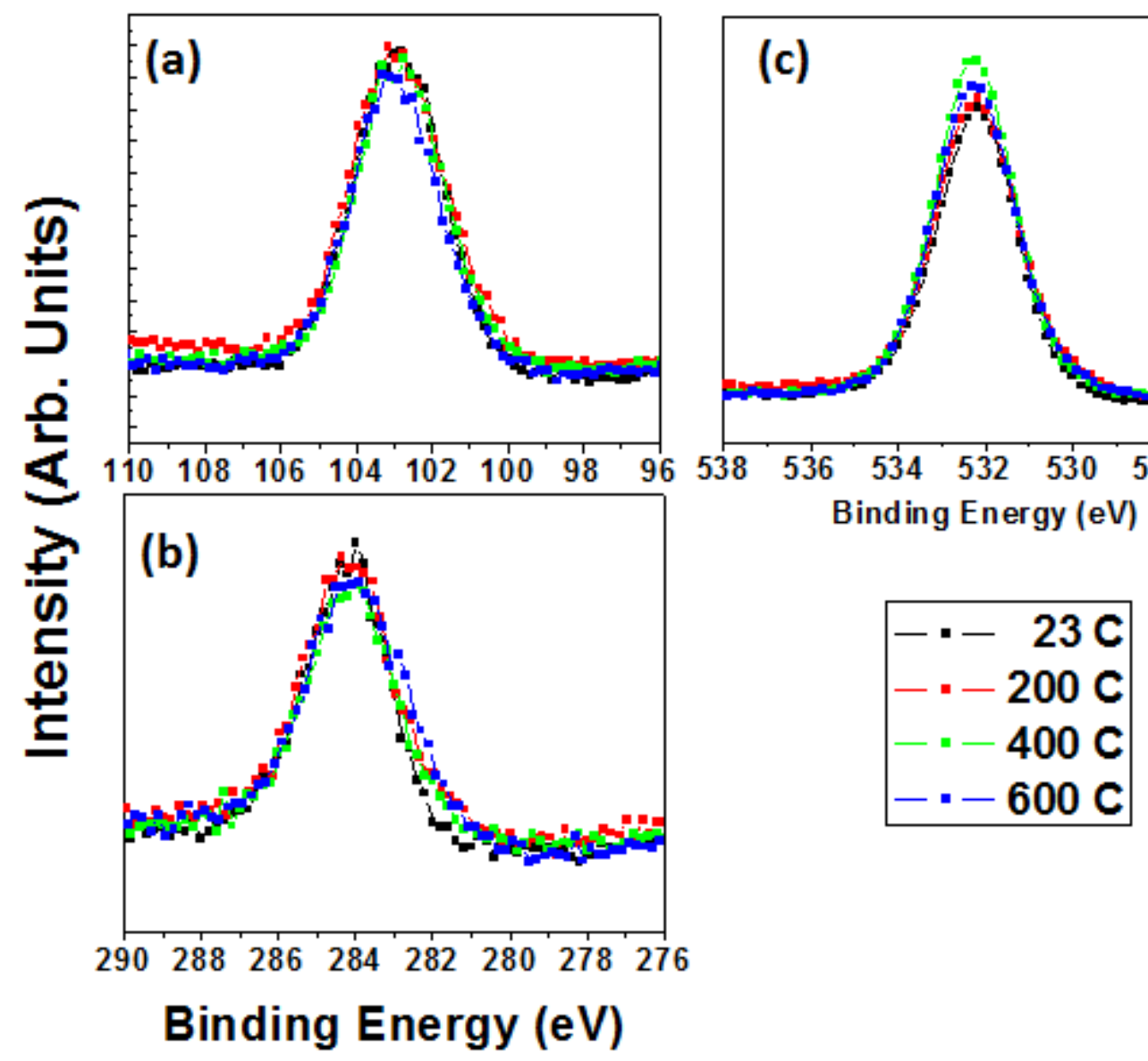

Figure 11 - The core level photoemission spectra for (a) Si 2p, (b) C 1s and (c) O 1s electronic orbital as a function of $\mathrm{He}^{+}$hot irradiation obtained for the $\mathrm{Si}-\mathrm{O}-\mathrm{C}$ alloy synthesized with a cosputtering rate of 1:1. Charging effects were referenced to the expected $\mathrm{O} 1 \mathrm{~s}$ signal. Photoelectrons were collected normal to the surface. The spectra correspond to that after the $5^{\text {th }}$ $\mathrm{Ar}^{+}$sputtered cycle. 


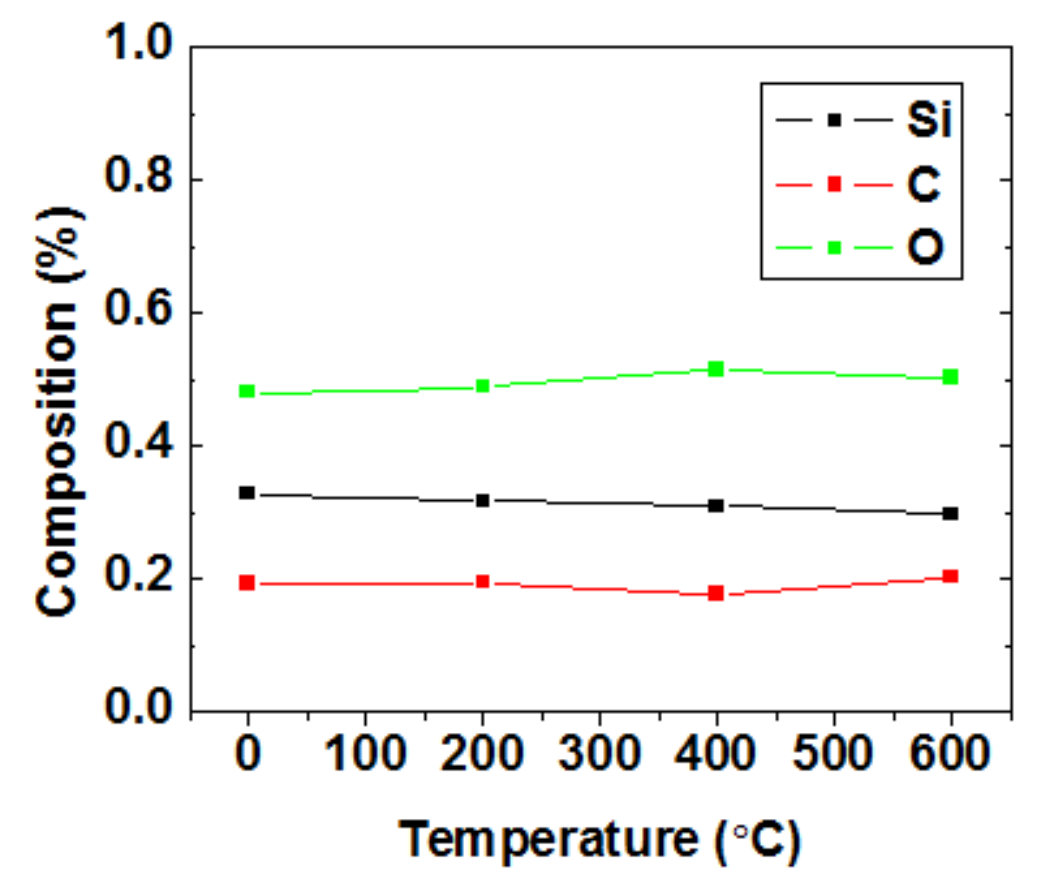

Figure 12 - The atomic composition obtained from the core level photoemission spectra for the $\mathrm{Si}-\mathrm{O}-\mathrm{C}$ alloy synthesized with a co-sputtering rate of $1: 1$ as a function of hot $\mathrm{He}^{+}$irradiation.

\section{Conclusion}

Composition engineered $\mathrm{Si}-\mathrm{O}-\mathrm{C}$ alloys were synthesized via co-sputtering growth using a $\mathrm{SiC}$ and $\mathrm{SiO}_{2}$ target. Diffractometry measurements provide evidence of thermal stability of the alloy up to $1000^{\circ}$ and annealing cycle of 2.0 hours. The shift in the amorphous peak to a higher diffraction angle suggest that the alloy undergo a small structural relaxation, possibly due to defects or unstable bonds from an thermally deprive growth. The retention of the alloy amorphousness was also confirmed under the chosen irradiation doses. X-ray photoelectron spectroscopy provides evidence of a structure relaxation as well, based on the charging observed in the alloys. XPS also provides evidence of a single phase alloy. The chemical state and composition preservation was also confirmed for both annealed and ion irradiated samples. The study suggests that the amorphous $\mathrm{Si}-\mathrm{O}-\mathrm{C}$ alloy may belong to a group of radiation tolerant materials. 


\section{Acknowledgement}

We acknowledge financial support from the DoE Office of Nuclear Energy, Nuclear Energy

Enabling Technologies, award DE-NE0000533. The work was carried out in part in the Central

Facilities of the Nebraska Center for Materials and Nanoscience, which is supported by the Nebraska Research Initiative. 


\section{References}

${ }^{1}$ J. B. Gibson, A. N. Goland, M. Milgram, and G. H. Vineyard, "Dynamics of Radiation Damage",Phys. Rev. 120, 1229 - Published 15 November 1960

2 B.N. Singh, "On the influence of grain boundaries on void growth", Phil. Mag., Volume 28, Issue 6, 1973 pages 1409-1413

${ }^{3}$ B.N. Singh, S.J. Zinkle, Defect accumulation in pure fcc metals in the transient regime: a review, Journal of Nuclear Materials, Volume 206, Issues 2-3, 2 November 1993, Pages 212-229

${ }^{4}$ Samaras, M., et al., Computer simulation of displacement cascades in nanocrystalline Ni. Physical Review Letters, 2002. 88 (12).

5 Bai, X.M., et al., Efficient Annealing of Radiation Damage Near Grain Boundaries via Interstitial Emission. Science, 2010. 327(5973): p. 1631-1634.

6 R. S. Averback, "Defects and Diffusion in Amorphous Alloys", MRS Bulletin, Volume 16, Issue 11, November 1991, pp 47-52

${ }^{7}$ R. S. Averback and H. Hahn, Physical Review B 37 (1988) 10383.

${ }^{8}$ P. J. Grundy, S. F. H. Parker, and G. A. Jones, Nuclear Instruments and Methods

${ }^{9}$ S. G. Mayr, Y. Ashkenazy, K. Albe, and R. S. Averback, Physical Review Letters 90 (2003) 055505.

${ }^{10}$ H. Trinkaus, Journal of Nuclear Materials 223 (1995) 196.

${ }^{11}$ P. Chaudhari, F. Spaepen, and P. J. Steinhardt, in Glassy Metals (H. Beck and H. J. Guntherodt, eds.), Springer-Verlag, Berlin, 1983, p. 127.

${ }^{12}$ H. Zhang and C.G. Pantano, J. of American Ceramic Soc., 73[4], pp. 958-963,1990.

${ }^{13}$ C.G. Pantano, A. Singh and H. Zhang, J. of Sol-Gel Science and Technology, 14, pp. 7-25, 1999

${ }^{14}$ Joseph V. Ryan and C. G. Pantano "Synthesis and characterization of inorganic silicon oxycarbide glass thin films by reactive rf-magnetron sputtering",J. Vac. Sci. Technol. A 25, 153 (2007)

15 Ping Du, Xiaoning Wang, I.-Kuan Lin, Xin Zhang, Effects of composition and thermal annealing on the mechanical properties of silicon oxycarbide films, Sensors and Actuators A: Physical, Volume 176, April 2012, Pages 90-98 
16 J.F. Ziegler, J.P. Biersack, and M.D. Ziegler, SRIM: The Stopping and Range of Ions in Matter, SRIM Co., Chester Maryland, 2008

17 G.S. Was, J.T. Busby, T. Allen, E.A. Kenik, A. Jenssen, S.M. Bruemmer, J. Gan, A.D. Edwards, P.M. Scott, and P.L. Andresen, J. Nucl. Mater. 300 (2002) 198

18 Eunjeong Jeona, Haengman Kima and Jondo Yuna, "Preparation of silicon oxycarbide amorphous ceramics from polymer precursors and the characterization of their high temperature stability", Journal of Ceramic Processing Research. Vol. 13, No. 3, pp. 239 242 (2012)

${ }^{19}$ Gian Domenico Sorarù, Daniel Suttor, "High Temperature Stability of Sol-Gel-Derived SiOC Glasses",Journal of Sol-Gel Science and Technology, March 1999, Volume 14, Issue 1, pp 69-74

${ }^{20}$ C. Virojanadaraa, P.-A. Glansa, L.I. Johanssona, Th. Eickhoffb, W. Drubec, "High energy photoemission investigations of $\mathrm{SiO}_{2} / \mathrm{SiC}$ samples", Applied Surface Science 172 (2001) $253 \pm 259$

21 Onneby, C. and Pantano, C. G.,"Silicon oxycarbide formation on SiC surfaces and at the $\mathrm{SiC} / \mathrm{SiO} 2$ interface", Journal of Vacuum Science \& Technology A, 15, 1597-1602 (1997)

${ }^{22}$ R. J. P. Corriu, D. Leclercq, P. H. Mutin, A. Vioux, "Preparation and structure of silicon oxycarbide glasses derived from polysiloxane precursors", Journal of Sol-Gel Science and Technology 1997, Volume 8, Issue 1-3, pp 327-330

${ }^{23}$ Gian Domenico Sorarù, Gennaro D'Andrea, Antonella Glisenti, XPS characterization of gelderived silicon oxycarbide glasses, Materials Letters, Volume 27, Issues 1-2, May 1996, Pages $1-5$

24 B. J. Tielsch and J.E. Fulghum, "Differential Charging in XPS. Part I: Demonstration of Lateral Charging in a Bulk Insulator Using Imaging XPS, Surface and Interfaces Analysis, Vol. 24, 422-427 (1996)

${ }^{25}$ S. Suzer, "Differential Charging in X-Ray Photoelectron Spectroscopy: A nuisance or a useful tool?", Ana. Chem., Vol. 75, 7026-7029 (2003)

26 Juan A. Colón Santana, Pan Liu, X. Wang, J. Tang, S.R. McHale, J.W. McClory, J.C. Petrosky, J. Wu, A. Rivera, R. Palai, Ya. B. Losovjy, and P.A. Dowben, "The local metallicity of gadolinium doped compound semiconductors", J. Phys.: Condens. Matter 24 (2012) 445801

27 Juan A. Colón Santana, Joonhee M. An, Ning Wu, Kirill Belashchenko, X. Wang, P. Liu, Jinke Tang, Yaroslav Losovyj, I.N. Yakovkin, and P.A. Dowben, "Altering the Oxide with Gadolinium Doping”, Phys. Rev. B 85, 014406 (2012) 
${ }^{28}$ Marcio Luis Ferreira Nascimento, "Correlation between Conductivity and Free Volume in Rubidium and Cesium Silicate Glasses", Brazilian Journal of Physics, vol. 37, no. 2A, June, 2007

${ }^{29}$ N. Hecking, K.F. Heidemann, E. Te Kaat, Model of temperature dependent defect interaction and amorphization in crystalline silicon during ion irradiation, Nuclear Instruments and Methods in Physics Research Section B: Beam Interactions with Materials and Atoms, Volume 15, Issues 1-6, 1 April 1986, Pages 760-764

${ }^{30}$ W.J. Weber, L.M. Wang, The temperature dependence of ion-beam-induced amorphization in $\beta-\mathrm{SiC}$, Nuclear Instruments and Methods in Physics Research Section B: Beam Interactions with Materials and Atoms, Volume 106, Issues 1-4, 2 December 1995, Pages 298-302

${ }^{31}$ L.Q. Zhang, C.H. Zhang, J. Gou, L.H. Han, Y.T. Yang, Y.M. Sun, Y.F. Jin, "PL and XPS study of radiation damage created by various slow highly charged heavy ions on GaN epitaxial layers", Nuclear Instruments and Methods in Physics Research B 269 (2011) 2835-2839 